\title{
Application of Strapdown Inertial Navigation Technology in Measurement
}

\author{
Feifei Wu \\ Shanghai University of \\ Engineering Science, China.
}

\author{
Zhengfeng Cao \\ Shanghai University of \\ Engineering Science, China.
}

\author{
Minglai Yang* \\ Shanghai University of Applied \\ Sciences, \\ Shanghai Advanced Research \\ Institute, Chinese Academy of \\ Sciences, China
}

\begin{abstract}
In this paper, combining the domestic and foreign research results, the application of strapdown inertial navigation technology in the measurement is summarized.First the basic principle of strapdown inertial navigation system are reviewed, and then discusses the application status of strapdown inertial navigation technology in military and civilian areas of measurement. Finally, the application of the strapdown inertial navigation technology development and the future outlook
\end{abstract}

\section{Keywords}

Strapdown inertial navigation, inertial measurement, aviation, track test

\section{INTRODUCTION}

Inertial measurement also called inertial geodesy, is a new technology which is based on the inertial technology, at the same time, it can obtain many kinds of geodetic data (such as latitude, longitude, elevation, azimuth, gravity anomaly and gravity line). Its principle is through the inertial components feeling carrier acceleration in the process of movement, carry on the two integral of acceleration by computer, can be concluded that the carrier in the space position change, etc. Due to its presence, to geodesy and rapid positioning provides a new prospect.

The function of the inertial measurement system is to measure the $3 \mathrm{D}$ coordinates of the carrier in a reference frame. Therefore, it must establish a reference coordinate system. There are two ways to provide reference frame. One is to realize the method by computer. The inertial element is directly mounted on the carrier, and the system is not required for the platform. Another kind of platform type measurement system, which is realized by using the platform system in physical way. The accelerometer is mounted on the platform, regardless of how the direction of the carrier is changed, and the orientation of the platform in the inertial space will remain consistent or consistent. As a desktop inertial navigation systems are compared, the strapdown inertial navigation system is abolished the mechanical platform, replaced by the navigation computer to establish a mathematical platform to replace the platform inertial navigation system in electrical and mechanical platform entity, complete function of mechanical platform[1].

\subsection{Characteristics of SINS}

(1) Because the IMU directly and fixedly connected on the carrier, so they measure is along the carrier coordinates of each axis inertial linear acceleration and around the carrier coordinates of each axis rotation rate.

(2) Get rid of the navigation platform, the size, weight and cost of the whole system is greatly reduced, improving reliability.

(3) Inertial instrument is convenient for installation and maintenance, also easy to replacement.

(4) Inertial instrument is easy to use redundant configuration, improve the system performance and reliability.

(5) Inertial instrument fixed carrier, directly from the vibration and impact of carrier, poor working conditions.

(6) Gyroscope of the platform system is installed on the platform, and can be compared with the acceleration of gravity and the earth rotation angular velocity of arbitrary orientation to test, to facilitate calibration. While the gyro of the strapdown inertial navigation system does not have this condition, the installed calibration is difficult, and the parameters of the strapdown gyroscope has higher stability.

(7) In the strapdown inertial navigation system, the computer calculation is much larger than platform inertial navigation system, the computer word length and speed requirements are much higher.

\subsection{Coordinate transformation}

In strapdown attitude algorithm, it mainly involves two coordinate system, the geographic coordinate system (or navigation coordinate system)and the carrier coordinate system[2].

In the Geographic coordinate system $\mathrm{OX}_{\mathrm{t}} \mathrm{Y}_{\mathrm{t}} \mathrm{Z}_{\mathrm{t}}$ :

$\mathrm{OX}_{\mathrm{t}}$ axis--in the local level refers to the east.

$\mathrm{OY}_{\mathrm{t}}$ axis--in the local level refers to the north

$\mathrm{OZ}_{\mathrm{t}}$ axis--along the local vertical direction point to the zenith.

In the carrier coordinate system $\mathrm{OX}_{\mathrm{b}} \mathrm{Y}_{\mathrm{b}} \mathrm{Z}_{\mathrm{b}}$ :

$\mathrm{OY}_{\mathrm{b}}$ axis--along the longitudinal axis of the carrier, pointing to the former.

$\mathrm{OX}_{\mathrm{b}}$ axis--along the horizontal axis of the carrier,pointing to the right .

$\mathrm{OZ}_{\mathrm{b}}$ axis--is perpendicular to the plane, and the vertical axis of the carrier is pointed upward. 
For the geographic coordinate system and the carrier coordinate system,their three axes both constitute Cartesian coordinates.Carrier coordinate system relative to the local geographic coordinate system can be expressed by the attitude angle, and realize the transformation of the vector coordinate system to the geographic coordinate system can be divided into three rotation in the following order[3].

First, $\mathrm{OX}_{\mathrm{t}} \mathrm{Y}_{\mathrm{t}} \mathrm{Z}_{\mathrm{t}}$ coordinate system turn $h$ degrees around the $\mathrm{Z}_{\mathrm{t}}$ axis,obtain $\mathrm{OX}_{\mathrm{b} 1} \mathrm{Y}_{\mathrm{b} 1} \mathrm{Z}_{\mathrm{t}}$ coordinate system.Next ,turn $\phi$ degrees around the $\mathrm{X}_{\mathrm{b} 1}$ axis, obtain $\mathrm{OX}_{\mathrm{b} 1} \mathrm{Y}_{\mathrm{b}} \mathrm{Z}_{\mathrm{b} 2}$ coordinate system.Finally,continue to turn $\theta$ degrees around the $\mathrm{Y}_{\mathrm{b}}$ axis, so then it can obtain $\mathrm{OX}_{\mathrm{b}} \mathrm{Y}_{\mathrm{b}} \mathrm{Z}_{\mathrm{b}}$ coordinate system.

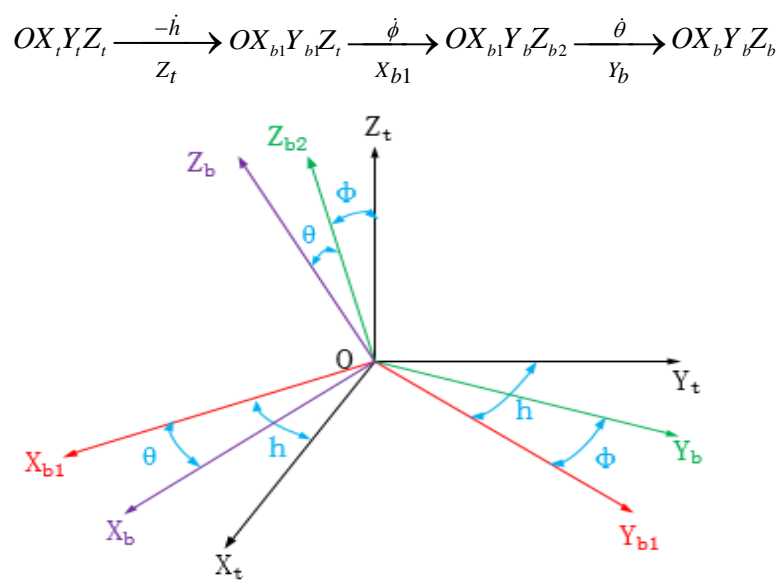

Fig 1: Coordinate transformation relation

\subsection{The working principle of SINS}

Strapdown inertial navigation system is composed of IMU(Inertial Measuring Unit), navigation computer and navigation display device.Figure 2 is the principle of its structure. The combination of accelerometer and gyroscope is known as inertial combination. structure. The combination of accelerometer and gyroscope is known as inertial combination.

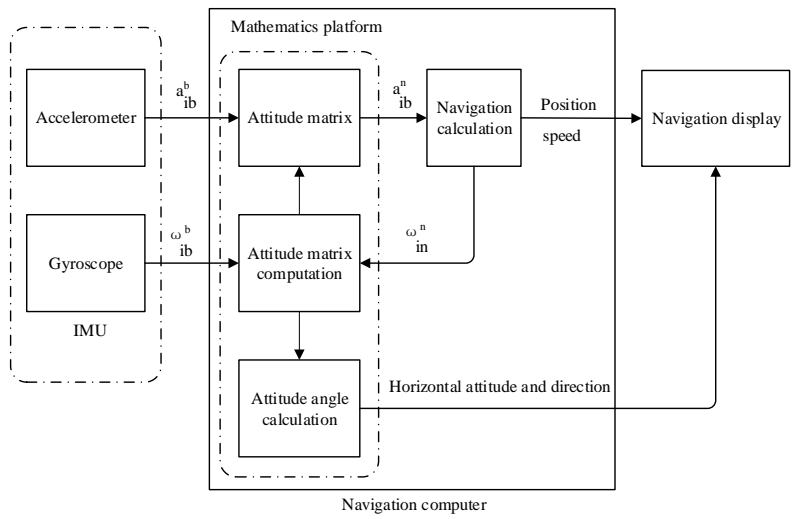

Fig 2: Schematic diagram of SINS

The acceleration of each axis relative to the inertial space is measured by the accelerometer, and the attitude matrix is updated with the angular velocity information of the gyro output,thus transform the acceleration component to the navigation coordinate system by the attitude matrix, and then, the motion information of the vector in 3D space can be obtained by the integral operation.

\section{APPLICATION OF SIN TECHNOLOGY IN MEASUREMENT}

Inertial technology involving materials,physics,precision control,mathematics, mechanics, electronic,computer,testing technology,and so on.Setting light,electricity,machine and other high-tech as a whole,is widely used in the fields of aerospace,marine,transportation,surveying,aviation,medical, agriculture,robot and consumption,it is a measure of an important indicator of a country's scientific and technological level and national defense strength[4].

These are the inherent disadvantages of inertial devices.Firstly,the initial self alignment problem of the strapdown inertial system when in the dynamic environment[5].Secondly,the navigation error accumulated over time, especially when the high precision and long time navigation,the reliability, accuracy and holding time of the inertial system are higher .In spite of the inherent shortcomings of the inertial navigation system,it has many advantages, therefore,the strapdown inertial navigation system have been widely used in all kinds of civil aircraft, rockets, cargo aircraft and all kinds of military aircraft, tactical missiles weapon systems .

\subsection{Military affairs}

\subsubsection{Application of inertial technology in various} stages of artillery operation

In the preparation stage of the geodesic:the artillery must consider terrain conditions in the routine measurement,and consider the atmospheric refraction and sidereal time in the observation.But in inertial measurement, there is no need to consider these issues.The outstanding characteristic of the inertial measurement is the all-weather, the independent work, the measurement process is only dependent on the function of the instrument itself.This feature is very much in line with the work of the artillery to measure the all-weather security tasks, and can be completed in the complex environment and strong electronic interference when using other ways to complete the task is difficult .

In a artillery-pointing stage:Artillery target must be conducted before shooting, the so-called aim, is to give the artillery pipe an accurate direction.In the conventional method, the initial position and the relative distance and direction of the target relative to the launch tube are determined,then the aim is to be aimed a by calculating. And through the principle of inertial navigation system,it can get initial shooting, shooting angle and roll angle by the initial alignment of the inertial system, so as to accurately draw artillery initial value.And the inertial system is sensitive to the change of the attitude of the transmitter, so as to ensure that the transmitting tube has the right direction, and the process of "aiming at the navigation" is completed[6].

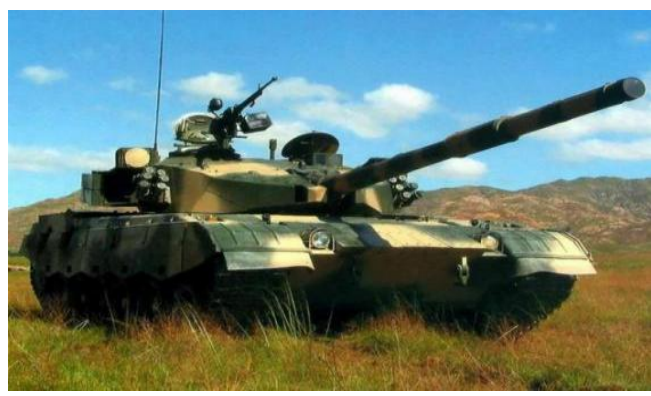

Fig 3: Artillery 


\subsubsection{Aviation}

The development of the aerospace inertial navigation system is related to the development of military aircraft. With the first generation airborne inertial navigation system are equipped with military aircraft of China in twentieth Century, the application and development level of China's aviation inertial technology has been greatly improved.At the same time, based on the dynamic tuning gyro strapdown inertial navigation system as the core, integrated satellite, doppler radar and magnetic sensors and other information, the use of redundancy configuration and multi sensor information fusion technology based integrated navigation system has formed a series of products, should be used in the field of low precision.

In recent years, with the continuous improvement of the level of laser gyro production and the continuous progress of the technology of strapdown inertial system,laser strapdown inertial navigation system has been finalized and started to form the equipment products, with its short preparation time, quick response ability, high precision of navigation, it has become the first choice for the new model development and the old type transformation.

\subsection{Civilian affairs}

\subsubsection{Communication system}

Emergency communication system as emergency command central nervous, in a variety of rescue events it can be timely and accurately transmit first-hand information, to ensure that the emergency command center, linkage platform and the scene communication open[7].

Satcom on the move is at the core of emergency communication industry equipment,is mainly composed of the antenna,feed systems,rotating platform,information feedback system,information collection and processing system,tracking control system,communication system,etc..Satcom on the move, because it has strong maneuverability,obvious advantages, in mobile communication,etc.,make it become the primary choice of communication in the face of disaster.

The strapdown inertial navigation system can be used in the Satcom on the move antenna control system, is a good way to solve the special requirements of the satellite antenna tracking system:on the one hand is that the antenna is required to respond quickly, and the target of the satellite can be quickly completed after the bridge, trees, buildings, mountains and tunnel lights shade, and on the other hand is to track the satellite target accurately and automatically,ensure the communication quality when the carrier is violently disturbed[8].Strapdown inertial navigation system provides the car body posture and heading information, can guide the satellite antenna pointing to the target quickly, when satellites within half power beam width of the antenna electrical axis (the range is usually $1^{\circ}$ ), satellite antenna control system automatically capture the satellite target and enter the high precision autonomous tracking state according to the information maximization principle, thus greatly shorten the capture time[9]\&[10].

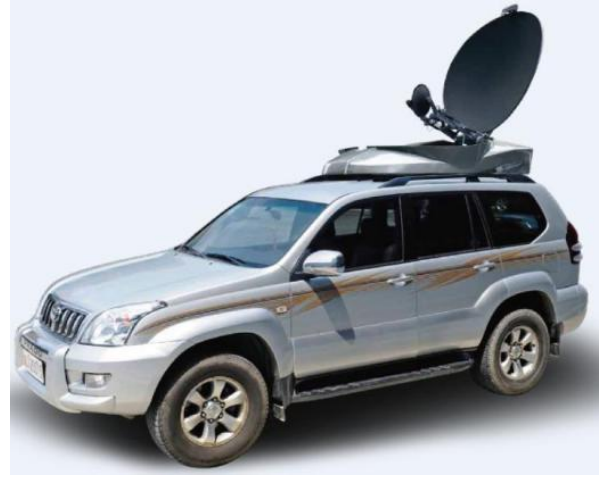

Fig 4: SatCom Land-mobile

\subsubsection{Track inspection}

With the development of the railway transportation routes in the large and medium-sized cities, the urban rail transit has entered a period of vigorous development.For railway and urban rail transit, track is the basis of the train running, so the track state is the direct factor that affects the safe operation of the train. With the large increase train speed and the rapid development of urban rail transit, high speed, high density of traffic environment cause track a huge load, the manual maintenance of busy trunk highway is increasingly not adapted to the reality,in order to ensure the safety and comfort of the passengers, high quality and high efficiency of the track maintenance has become an inevitable requirement for the maintenance of the railway and urban transportation.

Track inspection car based on inertial technology can track the railway regularly, judging whether the track is deformed or lost, and whether is foundation settlement.At the same time, the railway,subway,urban light rail and other fields need track inspection system,so the application of inertial technology products has broad prospects[11].For example, the Laserail track measuring system developed by the American ImageMap company adopts laser imaging and high speed image processing technology, the system includes two fiber optic gyro and two accelerometer and its analog processing board, 4 lasers, 10 cameras, etc.,can measure rail gauge,curvature,twist of track,superelevation,the rail top and side wear ,etc.[12]\&[13]

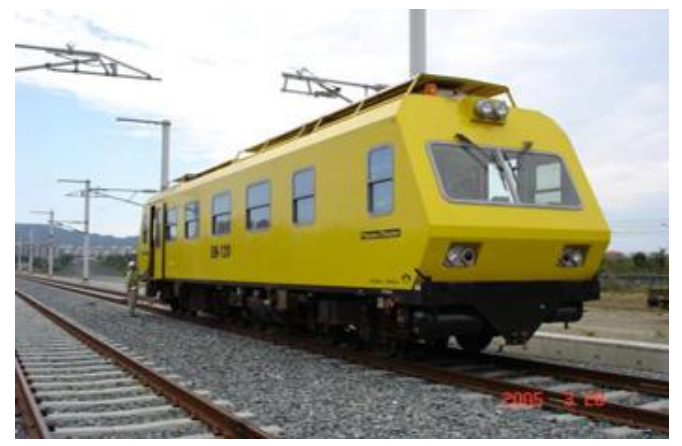

Fig 5: Track recording car

\subsubsection{Unmanned aerial vehicle}

The application of inertial technology in unmanned aerial vehicle is mainly based on the new type of inertial device and inertial navigation technology to provide accurate speed, position, attitude and so on,so as to achieve its accurate navigation and positioning. At present, the application of UAV in military field is the most mature.Also with the people of UAV cognition degree deepens, the surveying, mapping and remote sensing, edge Haiphong, forest fire prevention, 
emergency relief, police law enforcement and other civilian areas showing rapid development momentum.

\section{CONCLUSION}

The development of inertial technology in China has been for more than 60 years of history, has experienced from scratch, from weak to strong, from backwardness to advanced development, has created a series of brilliant achievements.At present, inertial technology has been widely used in the fields of geodetic survey,oil drilling, electronic transportation, automobile safety and consumer electronics.Based on this, the application of the strapdown inertial navigation technology in the measurement will become more extensive with the development of inertial technology.Based on the SINS,AHRS, GPS and magnetometer, altimeter, odometer integrated navigation system will appear in large quantities, used not only in the weapon system but also in civil industry.Bionic technology is likely to be added to the design concept of integrated navigation system. The next generation of integrated navigation systems will develop in the direction of the intelligent information perception and decision making system with the function of prediction[14].

\section{REFERENCES}
[1] Zhongsu,Qing Li,Kuangzhen Li,et al..Inertial Technology[M].BeiJing:National Defence Industry Press,2010.12,pp.142.

[2] Yanjuan Yang.Key techniques of strapdown inertial navigation system[D].Harbin Engineering University,2001.

[3] Wenfa Zhu.Research on dynamic detection method of track line state based on Strapdown Inertial Technology[D].Shanghai University Of Engineering Science, 2011.

[4] King A D.Inertial navigation-forty years of evolution. GEC Review,1998,13(3),pp.1-15.

[5] XiaoguangZhang.Three-dimensional function conditional extremum's sufficient conditions[J].Journal of Heilongjiang Institute of Science \& Technology,2002,12(3),pp.49-50.

[6] Zhenghua Wu,Qiang Chang,Bolong Wu,et al..Introduction to the application of inertial technology in artillery combat $[\mathrm{J}]$.Science and technology innovation herald,2011,(5),pp.245-245.

[7] Rong Zhang.Application of inertial technology in military and civilian market[J]. Digital communication world,2013,08,pp.14-19.

[8] Xiaogang RUA,Hongwu WANG.The technique andapplication of satellite communication antenna in motion[J].Satellite And Network,2006,6(3),pp.34-37.

[9] Rongxia DONG.Application of FOG strapdown attitude and heading reference system in-motion satellite communication[J].Electronic Design Engineering,2010,18(5),pp.76-77.

[10] Chunhua Yin,Guohua Lu,Laiding Zhao.Servo system of ship borne on board based on the control of Brushless DC motor[J].Journal of Chongqing University of Posts and Telecommunications:Natural Science Edition,2009,21(1),pp.87—91.

[11] Rong Zhang.Application of inertial technology in military and civilian market[J]. Digital communication world,2013,08,pp.14-19.

[12] Assembly of international communication materials for train[R].Ministry of Railways,2005.

[13] Yu Liu.Research on the full face detection system of vehicle type line[D].BeiJing:Degree paper of Beijing Jiaotong University,2010.pp.2-3.

[14] Zhiping Liu,Zonghu Hang.Present situation and developing trend of the technology of the optical strapdown inertial navigation system in foreign countries[J].Space control,2012,05,pp.94-99. 